\title{
Bottom-charmed meson spectrum from a QCD approach based on the Tamm-Dancoff approximation
}

\author{
L. M. Abreu $\odot^{*}$ \\ Instituto de Física, Universidade Federal da Bahia, Campus Universitário de Ondina, \\ Salvador, Bahia 40170-115, Brazil \\ F. M. da Costa Júnior $\oplus^{\dagger}$ \\ Instituto de Física, Universidade Federal da Bahia, Campus Universitário de Ondina, \\ Salvador, Bahia 40170-115, Brazil \\ and Instituto Federal do Sertão Pernambucano, Petrolina, Pernambuco 56316-686, Brazil
}

A. G. Favero $\oplus^{\ddagger}$

Department of Physics, McGill University, Montréal, Québec H3A 2T8, Canada

(Received 20 May 2020; accepted 13 July 2020; published 5 August 2020)

\begin{abstract}
The bottom-charmed meson spectrum is studied via an effective version of the Coulomb gauge QCD Hamiltonian. The Tamm-Dancoff approximation is employed to estimate the energies of the low-lying and radial-excited $B_{c}$ states with quantum numbers $J^{P}=0^{-}, 0^{+}, 1^{-}, 1^{+}, 2^{+}, 2^{-}$. In particular, we analyze the effects of incorporating an effective transverse hyperfine interaction and spin mixing. The Regge trajectories and hyperfine splitting of both $S$ - and $P$-wave states are also examined. The numerical results are compared with available experimental data and the theoretical predictions of other models.
\end{abstract}

DOI: 10.1103/PhysRevD.102.034002

\section{INTRODUCTION}

Despite the enormous experimental developments in heavy-hadron physics in recent decades, bottom-charmed $\left(B_{c}\right)$ spectroscopy remains much less known than the charmonium and bottomonium sectors. The reason for this comes from the fact that, since $B_{c}$ is a quarkonium bound state consisting of heavy quarks with different flavors $(c \bar{b}$ or $b \bar{c})$, the production mechanism demands factories of $c \bar{c}$ and $b \bar{b}$ pairs, which results in a small production rate. On the other hand, the different quark-flavor content denies its annihilation into gluons, engendering uniquely weak decays for the pseudoscalar ground state $B_{c}(1 S)$ and hadronic or radiative transitions for excited states which are below the strong-decay $B D$ threshold. These aspects suggest that $B_{c}$ states are more stable than their analogs in charmonium and bottomonium families, and it is therefore fairly important to study heavy-quark dynamics to understand the dynamics of the strong interaction in a deeper level.

\footnotetext{
*luciano.abreu@ufba.br

francisco.miguel@ifsertao-pe.edu.br

*aline.favero@mail.mcgill.ca
}

Published by the American Physical Society under the terms of the Creative Commons Attribution 4.0 International license. Further distribution of this work must maintain attribution to the author(s) and the published article's title, journal citation, and DOI. Funded by SCOAP.
The first observation of the $B_{c}$ meson was performed by the CDF Collaboration more than two decades ago [1], with the detection of the pseudoscalar ground state $B_{c}(1 S)^{+}$. It was confirmed later by other collaborations $[2,3]$ and is the only state considered an established particle with recognized quantum numbers, according to Particle Data Group (PDG) [4], with mass $(6274.9 \pm 0.8) \mathrm{MeV}$. The other state present in PDG, with mass $(6871.0 \pm 1.7) \mathrm{MeV}$ and identified as $B_{c}(2 S)^{+}$, has not had its quantum numbers confirmed. This is due to the controversy raised by the results from the ATLAS [5], CMS [6], and LHCb [7] collaborations. ATLAS [5] reported the mass $(6842 \pm 4 \pm 5) \mathrm{MeV}$ of an observed state, which is consistent with the first radially excited pseudoscalar, while very recently CMS [6] and $\mathrm{LHCb}$ [7] detected two signals consistent with the $2^{1} S_{0}$ and $2^{3} S_{1}$ states: for the $2^{1} S_{0}$ state, the LHCb and CMS found the mass as, respectively, $(6872.1 \pm 1.3 \pm 0.1 \pm 0.8)$ and $(6871.0 \pm 1.2 \pm 0.8 \pm 0.8) \mathrm{MeV}$. Besides, for the $2^{3} S_{1}$ state, LHCb obtained the mass $(6841 \pm 0.6 \pm 0.1 \pm 0.8) \mathrm{MeV}$, whereas CMS observed the mass difference $m\left[B_{c}\left(2^{1} S_{0}\right)\right]-$ $m\left[B_{c}^{*}\left(2^{3} S_{1}\right)\right]=(29 \pm 1.5 \pm 0.7) \mathrm{MeV}$. Thus, there are two intriguing features of these reports. The first one is the apparent disagreement between the ATLAS and CMS and $\mathrm{LHCb}$ outcomes for the $B_{c}^{*}\left(2^{3} S_{1}\right)$ meson. One possible explanation is that the peak observed by ATLAS could be the superposition of the $B_{c}\left(2^{1} S_{0}\right)$ and $B_{c}^{*}\left(2^{3} S_{1}\right)$ states, quite narrowly spaced with respect to the resolution of the 
measurement. The second one is that the $B_{c}\left(2^{1} S_{0}\right)$ state emerges as heavier than the mass $B_{c}^{*}\left(2^{3} S_{1}\right)$, which is in conflict with theoretical estimations. The plausible justification is that the observed $B_{c}^{*}(2 S)$ peak has a mass lower than the true value, which remains unknown due to the impossibility of reconstruction of the low-energy photon emitted when $B_{c}^{*+} \rightarrow$ $B_{c}^{+} \gamma$ [6]. Hence, more observations about the $B_{c}$-meson family are expected in the near future to help get a detailed characterization of heavy meson spectroscopy.

On theoretical grounds, different perspectives have been taken to investigate the $B_{c}$-meson spectrum as well as to understand its properties. For example, work can be found in the context of nonrelativistic quark models [8-16], relativistic constituent quark models [17-22], quantum chromodynamics (QCD) sum rules [23-25], lattice QCD [26-28], and approaches to the Dyson-Schwinger and Bethe-Salpeter equations [29]. The point here is that this miscellany of distinct approaches produces a frame to be contrasted with available and future experimental results, which in the end makes compelling comprehension of the $B_{c}$ state phenomenology possible.

That being so, this paper intends to contribute to the discussion and characterization of the $B_{c}$-meson spectrum by employing a different formalism with respect to the analyses mentioned in the previous paragraph. The framework to be utilized is also known as the Coulomb gauge QCD model [30-47]. This formulation is based on the exact QCD Hamiltonian in the Coulomb gauge, which is replaced by an effective Hamiltonian where the original nonperturbative confining and hyperfine interactions can be rearranged into calculable effective potentials between color densities and currents. The current quark and gluon field operators are dressed via the Bogoliubov-Valatin method. This provides the possibility of using relativistic field theory and many-body techniques such as TammDancoff and random phase approximations. The vacuum is represented as a coherent BCS ground state with quark and gluon Cooper pairs (condensates), and the hadrons interpreted as quasiparticle excitations. This approach has been successfully applied to the description of properties of some types of light and heavy mesons, glueballs, gluelumps, hybrids, and tetraquarks [30-47]. These reports demonstrate that this model is efficient in retrieving the essential aspects of QCD with a minimal number of free parameters (current quark masses and dynamical constants) and yielding reasonable predictions.

Here we extend the range of applications of the Coulomb gauge QCD model by studying the basic features of $B_{c}$ mesons within a unified scheme. The interactions between quarks and antiquarks will be treated through an improved confining potential and a transverse hyperfine interaction whose kernel is a Yukawa-type potential. Estimations for the energies of the low-lying and radial-excited $B_{c}$ states with quantum numbers $J^{P}=0^{-}, 0^{+}, 1^{-}, 1^{+}, 2^{+}, 2^{-}$are obtained. Also, the Regge trajectories are constructed, and a discussion about the hyperfine splittings of $S$ - and $P$-wave spectroscopy is done. The comparison of our results with other works is performed as well.

The paper is organized as follows. In Sec. II, we present the Coulomb gauge QCD model within the Tamm-Dancoff approximation. Section III is devoted to showing and analyzing the numerical calculations of the bottom-charmed meson spectrum, the Regge trajectories, and the hyperfine splittings. Concluding remarks are given in Sec. IV. In the Appendixes, we explicitly present the $B_{c}$-meson spin-orbital wave functions and kernels of the Tamm-Dancoff approximation (TDA) equation of motion used.

\section{THE MODEL}

Let us start by introducing the formalism to be used in the analysis of the $B_{c}$-meson spectrum. It is a Coulomb gauge QCD-inspired model whose effective Hamiltonian is given by $[32-38,41,42,46,47]$

$H_{\text {eff }}=\int d \mathbf{x} \Psi^{\dagger}(\mathbf{x})[-i \alpha \cdot \boldsymbol{\nabla}+\beta m] \Psi(\mathbf{x})+H_{C}+H_{T}$,

where $\Psi$ and $m$ are the current quark field and mass, respectively. The terms $H_{C}$ and $H_{T}$ are the effective couplings associated with the Coulomb and quark hyperfine interactions, i.e.,

$$
\begin{aligned}
& H_{C}=-\frac{1}{2} \int d \mathbf{x} d \mathbf{y} \rho^{a}(\mathbf{x}) \hat{V}(|\mathbf{x}-\mathbf{y}|) \rho^{a}(\mathbf{y}), \\
& H_{T}=\frac{1}{2} \int d \mathbf{x} d \mathbf{y} J_{i}^{a}(\mathbf{x}) \hat{U}_{i j}(\mathbf{x}, \mathbf{y}) J_{j}^{a}(\mathbf{y}),
\end{aligned}
$$

where $\rho^{a}(\mathbf{x})=\Psi^{\dagger}(\mathbf{x}) T^{a} \Psi(\mathbf{x})$ are the color densities and $\mathbf{J}^{a}(\mathbf{x})=\Psi^{\dagger}(\mathbf{x}) \boldsymbol{\alpha} T^{a} \Psi(\mathbf{x})$ the quark color currents, with $T^{a}$ $(a=1,2, \ldots, 8)$ being the $S U_{c}(3)$ generators. In the equations above, the flavor indices are not explicitly displayed so as to simplify the notation. Also, it should be mentioned that pure gluonic contributions have been excluded due to the fact that our interest is limited to the $q \bar{q}$ states.

We write below the kernels of the effective couplings in Eq. (2) used in the calculations. For the Coulomb longitudinal interaction $H_{C}$, the kernel is assumed to be an improved confining potential based on Yang-Mills dynamics, which in momentum space is represented as [36]

$$
V(p)= \begin{cases}\left(-12.25 \frac{m_{g}^{1.93}}{p^{3.93}}\right), & p<m_{g}, \\ -\frac{8.07}{p^{2}} \frac{\ln \left(\frac{p^{2}}{m_{g}^{2}}+0.82\right)^{-0.62}}{\ln \left(\frac{p^{2}}{m_{g}^{2}}+1.41\right)^{0.8}}, & p>m_{g}\end{cases}
$$

where $m_{g}$ is a parameter. Although we are not directly dealing with dynamical gluons in our model, we interpret them as responsible for $V(p)$, obtained from a 
self-consistent method of the non-Abelian degrees of freedom in the presence of static quarks, as noticed by Szczepaniak and Swanson [33]. Viewed in this way, $m_{g}$ can be interpreted as a dynamical mass scale for the constituent gluons.

Turning to the term $H_{T}$, it is associated with the quark hyperfine interaction of type $\vec{\alpha} \cdot \vec{\alpha}$ from the second-order coupling between quarks and transverse gluons after integrating out gluonic degrees of freedom. In this sense, the effective transverse hyperfine potential carries the kernel $\hat{U}_{i j}$ which keeps the structure of the transverse gauge condition

$$
\hat{U}_{i j}(\mathbf{x}, \mathbf{y})=\left(\delta_{i j}-\frac{\nabla_{i} \nabla_{j}}{\nabla^{2}}\right)_{\mathbf{x}} \hat{U}(|\mathbf{x}-\mathbf{y}|),
$$

with $\hat{U}$ being chosen to mimic one-gluon exchange potential. Following the analysis done in Ref. [36], in which a Yukawa-type potential appears as the preferred one for reasonable meson descriptions, we choose

$$
U(p)=C_{h} \begin{cases}(-24.57) \frac{1}{p^{2}+m_{g}^{2}}, & p<m_{g}, \\ -\frac{8.07}{p^{2}} \frac{\ln \left(\frac{p^{2}}{m_{g}^{2}}+0.82\right)^{-0.62}}{\ln \left(\frac{p^{2}}{m_{g}^{2}}+1.41\right)^{0.8}}, & p>m_{g},\end{cases}
$$

with the constant $C_{h}$ standing for the global strength, and the factor $(-24.57)$ being determined by matching the high and low momentum ranges at the scale $m_{g}$.

Next, we apply an appropriate quark basis in which calculations for meson states are most conveniently made. Following the standard Bogoliubov-Valatin method (see, for example, Ref. [34]), we perform the Bogoliubov transformation from the current quark basis to an improved quasiparticle quark basis represented by quasiparticle and antiquasiparticle $B_{\lambda c}^{(\dagger)}(\mathbf{k}), D_{\lambda c}^{(\dagger)}(\mathbf{k})$ operators, which allows us to write the quark field as

$$
\begin{aligned}
\Psi(\mathbf{x})= & \sum_{\lambda i} \int \frac{d^{3} k}{(2 \pi)^{3}}\left[\mathcal{U}_{\lambda}(\mathbf{k}) B_{\lambda i}(\mathbf{k})\right. \\
& \left.+\mathcal{V}_{\lambda}(-\mathbf{k}) D_{\lambda i}^{\dagger}(-\mathbf{k})\right] e^{i \mathbf{k} \cdot \mathbf{x}} \hat{\boldsymbol{\epsilon}}_{i},
\end{aligned}
$$

where $\lambda$ and $i$ denote the helicity and color indices $(i=1$, $2,3)$, respectively; $\left\{\hat{\boldsymbol{\epsilon}}_{c}\right\}$ is the color vector basis; and $\mathcal{U}$ and $\mathcal{V}$ are Dirac spinors forming a complete basis,

$$
\begin{gathered}
\mathcal{U}_{\lambda}(\mathbf{k})=\frac{1}{\sqrt{2}}\left(\begin{array}{c}
\sqrt{1+\sin \phi(k)} \chi_{\lambda} \\
\sqrt{1-\sin \phi(k)} \boldsymbol{\sigma} \cdot \hat{\mathbf{k}} \chi_{\lambda}
\end{array}\right), \\
\mathcal{V}_{\lambda}(-\mathbf{k})=\frac{1}{\sqrt{2}}\left(\begin{array}{c}
-\sqrt{1-\sin \phi(k)} \boldsymbol{\sigma} \cdot \hat{\mathbf{k}} i \sigma_{2} \chi_{\lambda} \\
\sqrt{1+\sin \phi(k)} i \sigma_{2} \chi_{\lambda}
\end{array}\right),
\end{gathered}
$$

with $\chi_{\lambda}$ being the Pauli spinors.
The Bogoliubov angle $\phi(|\mathbf{k}|) \equiv \phi_{k}$ connecting the current and quasiparticle quark bases is obtained by the variational minimization of the quasiparticle vacuum energy $\delta\langle\Omega|H| \Omega\rangle=0$, yielding the gap equation

$k s_{k}-m c_{k}=\int_{0}^{\infty} \frac{q^{2}}{6 \pi^{2}}\left[s_{k} c_{q}\left(V_{1}+2 W_{0}\right)-s_{q} c_{k}\left(V_{0}+U_{0}\right)\right]$,

where the functions $s_{k} \equiv \sin \phi_{k}$ and $c_{k} \equiv \cos \phi_{k}$ are related to the running quark mass $M(k)$ through the relationship $M(k)=k \tan \phi_{k}$. We identify $M(k) \rightarrow m$ at high $k$, while at low $k$ the constituent quark mass is extracted, $M(0) \rightarrow \mathcal{M}$. The functions $V_{0}, V_{1}$ and $U_{0}$ denote angular integrals of longitudinal and transverse potentials in the form

$$
F_{n}(k, q) \equiv \int_{-1}^{1} d x x^{n} F(|\mathbf{k}-\mathbf{q}|),
$$

with $x=\hat{k} \cdot \hat{q}$, and the $W$ function is defined as

$$
W(|\mathbf{k}-\mathbf{q}|) \equiv U(|\mathbf{k}-\mathbf{q}|) \frac{x\left(k^{2}+q^{2}\right)-k q\left(1+x^{2}\right)}{|\mathbf{k}-\mathbf{q}|^{2}} .
$$

Also, the expectation value of the effective Hamiltonian with respect to the one-quasiparticle state $|q\rangle \equiv B_{\alpha c}^{\dagger}(\mathbf{k})|\Omega\rangle$ engenders the expression that can be identified as the selfenergy of the quasiparticle,

$$
\begin{aligned}
\epsilon_{k} & =\left\langle q\left|H_{\mathrm{eff}}\right| q\right\rangle \\
& =m s_{k}+k c_{k} \\
& -\int_{0}^{\infty} \frac{q^{2}}{6 \pi^{2}}\left[s_{k} s_{q}\left(V_{0}+2 U_{0}\right)+c_{k} c_{q}\left(V_{1}+W_{0}\right)\right] .
\end{aligned}
$$

It must be observed that a meson in this framework is supposed to be an excited state consisting of a bound state of the quasiparticle and antiquasiparticle. Then it is useful to introduce the meson creation operator in the TDA scheme, which is a bosonization method that has been revealed to be a good approximation for a large number of meson families, excluding only the case of the pions. Accordingly, the quasiparticle-antiquasiparticle operator is given by

$$
Q_{n J P}^{\dagger}=\sum_{\lambda \lambda^{\prime}} \int \frac{d \mathbf{k}}{(2 \pi)^{3}} \Psi_{\lambda \lambda^{\prime}}^{(n J P)}(\mathbf{k}) B_{\lambda}^{\dagger}(\mathbf{k}) D_{\lambda^{\prime}}^{\dagger}(-\mathbf{k}),
$$

where $\Psi_{\alpha \beta}^{(n J P)}$ means the wave function corresponding to an open-flavor meson state with total angular momentum $J$, parity $P$, and radial quantum number $n$ (we have omitted the color and flavor indices).

Now the method of calculating the energy levels of mesonic bound states can be expressed. The energies are 
obtained via the TDA equation of motion for an open-flavor meson, defined by

$$
\left\langle\Psi^{(n J P)}\left|\left[H_{\mathrm{eff}}, Q_{n J P}^{\dagger}\right]\right| \Omega\right\rangle=\left(E_{n J P}-E_{0}\right)\left\langle\Psi^{(n J P)}\left|Q_{n J P}^{\dagger}\right| \Omega\right\rangle .
$$

This equation can be recast into a more convenient form by profiting from the rotational invariance of $H_{\text {eff }}$ and constructing the wave functions via multiplication of Pauli $\sigma$ matrices by powers of orbital momentum $\hat{k}^{l}$ to get partial waves. Concerning the last procedure, we indicate to the reader the Appendix A of Ref. [46], in which the specific case of axial mesons is discussed. Notwithstanding, for completeness we express in detail the wave functions exploited in this work, which can be written as (again omitting the flavor indices)

$$
\Psi_{\lambda \lambda^{\prime}}^{(n J P)}(\mathbf{k})=\frac{\delta_{i j}^{(\text {color })}}{\sqrt{3}} R^{(n J P)}(k) \psi_{\lambda \lambda^{\prime}}^{(J P)}(\mathbf{k})
$$

where $R^{(n J P)}(k)$ is the radial wave function. $\psi_{\lambda \lambda^{\prime}}^{(J P)}(\mathbf{k})$ carries the angular-momentum dependence and assumes a distinct form according to the nature of the meson state described by the quantum numbers $L, S, J$, which specify the parity $P=(-1)^{L+1}$ and also the charge conjugation $C=(-1)^{L+S}$ if the quark and antiquark have opposite flavors (equal mass). These wave functions are given explicitly in Appendix A. After that, we perform the diagonalization of the effective Hamiltonian in the TDA representation, which is undertaken by the computation of the trace of spinor products coming from commutators on the left-hand side of Eq. (14). The final expression for the TDA equation of motion is

$$
\begin{aligned}
M_{n J P} R^{(n J P)}(k)= & \left(\epsilon_{k}^{b}+\epsilon_{k}^{c}\right) R^{(n J P)}(k) \\
& +\int_{0}^{\infty} \frac{q^{2} d q}{12 \pi^{2}} K^{(J P)}(k, q) R^{(n J P)}(q),
\end{aligned}
$$

where $M_{n J P} \equiv E_{n J P}-E_{0}$ is the energy of the $B_{c}$-meson state, $\epsilon_{k}^{b}\left(\epsilon_{k}^{c}\right)$ is the self-energy of the (anti)quasiparticle associated with the $b(c)$ quark, and $K^{(J P)}(k, q)$ is the kernel bearing the potential terms, which is dependent on the meson quantum numbers. We should remark that several versions of kernels are accessible in the literature, written using a different basis as well as distinct interaction terms. At this time, the tensor cases with both longitudinal and transverse potentials, however, are not available (at least to our knowledge). In view of these considerations, the relevant kernels obtained for the mesons described by the wave functions given by Eqs. (A1)-(A8) are expressed in Appendix B [Eqs. (B2)-(B8)].

As a final comment in this section, we must note that open-flavor mesons, like $B_{c}$ mesons, are not eigenstates of charge conjugation since they have a quark and antiquark with different flavors. Therefore, the total spin $(S)$ is no longer a good quantum number, and spin-singlet and spintriplet states with $J=L$ can mix. This is the case for the axial $\left(n^{3} P_{1}\right.$ and $\left.n^{1} P_{1}\right)$ and pseudotensor $\left(n^{3} D_{2}\right.$ and $\left.n^{1} D_{2}\right)$ states reported above. A simple mixing prescription for these $J=L$ states is

$$
\begin{aligned}
& \left|n L_{L}^{\prime}\right\rangle=\cos \theta_{n L}\left|n^{1} L_{L}\right\rangle+\sin \theta_{n L}\left|n^{3} L_{L}\right\rangle, \\
& \left|n L_{L}\right\rangle=-\sin \theta_{n L}\left|n^{1} L_{L}\right\rangle+\cos \theta_{n L}\left|n^{3} L_{L}\right\rangle,
\end{aligned}
$$

where $\theta_{n L}$ is the mixing angle and $n L_{L}^{\prime}, n L_{L}$ are the physical states. Supposing that the masses of $b$ and $c$ quarks satisfy the limit $m_{b} \gg m_{c}$, this leads to the extreme heavylight expression $\theta_{n L} \rightarrow \tan ^{-1} \sqrt{L /(L+1)}$, which gives $\theta_{n P} \rightarrow 35.3^{\circ}, \theta_{n D} \rightarrow 39.2^{\circ}$. Here we adopt the following relation between the masses of the $\left(n^{3} L_{L}-n^{1} L_{L}\right)$ and $\left(n L_{L}^{\prime}-n L_{L}\right)$ pairs [48]:

$$
\begin{aligned}
M\left(n L_{L}\right)= & M\left(n^{1} L_{L}\right) \cos ^{2} \theta_{n L}+M\left(n^{3} L_{L}\right) \sin ^{2} \theta_{n L}-\left[M\left(n^{3} L_{L}\right)\right. \\
& \left.-M\left(n^{1} L_{L}\right)\right] \frac{\sin ^{2} 2 \theta_{n L}}{2 \cos 2 \theta_{n L}}, \\
M\left(n L_{L}^{\prime}\right)= & M\left(n^{1} L_{L}\right) \sin ^{2} \theta_{n L}+M\left(n^{3} L_{L}\right) \cos ^{2} \theta_{n L}+\left[M\left(n^{3} L_{L}\right)\right. \\
& \left.-M\left(n^{1} L_{L}\right)\right] \frac{\sin ^{2} 2 \theta_{n L}}{2 \cos 2 \theta_{n L}} .
\end{aligned}
$$

\section{NUMERICAL RESULTS}

In this section, the results for the spectrum of the $B_{c}$ mesons are exhibited, generated with the model sketched out above. Briefly, the strategy consists of solving the gap equation [Eq. (9)] for each flavor to get the $k$-dependent gap angles $\phi_{k}^{b}$ and $\phi_{k}^{c}$; they supply the values of functions $c_{k(q)}^{b(c)}$ and $s_{k(q)}^{b(c)}$ to generate $M_{n J P}$ that numerically solve the TDA equation of motion in Eq. (16). The adoption in the calculations of kernels with interactions represented by an improved confining potential and a transverse Yukawatype potential playing the role of the exchange of a constituent gluon should be emphasized.

In the Coulomb gauge QCD model, the input parameters to be fitted to the experimental data are the dynamical mass of the constituent gluon $m_{g}$, the current quark masses of the $b$ and $c$ quarks, $m_{b}$ and $m_{c}$, and the magnitude of the transverse potential $C_{h}$. However, as discussed in the Introduction, data for the $B_{c}$-meson families are scarce at present, despite recent results from the ATLAS [5], CMS [6], and LHCb [7] collaborations. According to PDG [4], to date there have been two $B_{c}$ mesons observed. The ground pseudoscalar state is the only one considered as an established particle, with mass $M\left[B_{c}(1 S)^{+}\right] \approx(6274.9 \pm$ $0.8) \mathrm{MeV}$; the other one, with mass $(6871.0 \pm 1.7) \mathrm{MeV}$, is consistent with the first radially excited pseudoscalar, but 
quantum numbers are not confirmed. Nevertheless, it should also be mentioned that the ATLAS and LHCb collaborations reported the observations of peaks at $(6842 \pm 4 \pm 5)$ and $(6841 \pm 0.6 \pm 0.1 \pm 0.8) \mathrm{MeV}$, respectively, which are consistent with the $B_{c}^{*}\left(2^{3} S_{1}\right)$ state. Remarking that the goal here is to extract the basic picture of the $B_{c}$-meson spectrum, the values of the parameters $\left(m_{b}, m_{c}, m_{g}, C_{h}\right)$ are adjusted to reproduce approximately these reported states, in particular, the confirmed $B_{c}(1 S)^{+}$.

We start by showing in Table I the values of constituent quark masses $\mathcal{M}_{b, c}$ engendered by the current quark masses $m_{b}=4000 \mathrm{MeV}$ and $m_{c}=950 \mathrm{MeV}$ that are used as inputs in this subsection. The remaining parameters $C_{h}$ and $m_{g}$ are taken with different but close values so as to evaluate their impact on the constituent quark masses $\mathcal{M}_{b, c}$, extracted from the limit $M_{b, c}(k \rightarrow 0) \equiv \mathcal{M}_{b, c}$. They are chosen while obviously keeping in mind the range that better matches the physical states. It can be seen that the growth of $C_{h}$ and $m_{g}$ yields greater values of $\mathcal{M}_{b, c}$ because of the modification of the gap angles coming from solutions of the gap equation. We stress that the values of current and constituent quark masses are smaller than they are in some quark models due to the contributions from interaction potentials in gap equation (9) and in the self-energy $\epsilon_{k}^{b, c}$ [Eq. (12)]. For a detailed discussion, we refer the reader to Refs. [36,46]. In this regard, it deserves to be cited that very recent $\left(n_{f}=2+1+1\right)$-lattice QCD calculations obtained estimations for the charm quark mass by about 980 $995 \mathrm{MeV}$ [49,50], which are close to the one that we utilize.

For the sake of completeness, we briefly discuss the overall momentum dependence of the Bogoliubov angles for the different flavors obtained from the solutions of the gap equation [Eq. (9)]. To this end, in Fig. 1 the solutions $\phi_{k}^{b}$ and $\phi_{k}^{c}$ are plotted as a function of $k$. At higher values of $k$, the solutions exhibit a decreasing exponential behavior, with the $c$-flavor case experiencing a faster lessening. In particular, the obtention of $\phi_{k}^{b, c} \rightarrow 0$ in the limit $k \rightarrow \infty$

TABLE I. The constituent quark masses $\mathcal{M}_{b, c}$ engendered by the current quark masses $m_{b}=4000 \mathrm{MeV}$ and $m_{c}=950 \mathrm{MeV}$ used as inputs in this subsection. $\mathcal{M}_{b, c}$ are obtained from the gap angles $\phi_{k}^{b}$ and $\phi_{k}^{c}$ that solve the gap equation [Eq. (9)] through the relationship $\lim _{k \rightarrow 0} M_{b, c}(k)=\lim _{k \rightarrow 0} k \tan \phi_{k}^{b, c} \equiv \mathcal{M}_{b, c}$. The first column denotes the set of parameters $\left(m_{g}, C_{h}\right)$ used. All quantities are given in $\mathrm{MeV}$ except the value of $C_{h}$, which is adimensional.

\begin{tabular}{lcc}
\hline \hline Set $\left(m_{g}, C_{h}\right)$ & $\mathcal{M}_{c}$ & $\mathcal{M}_{b}$ \\
\hline I $(600,0.4)$ & 1208 & 4343 \\
II $(650,0.4)$ & 1222 & 4362 \\
III $(700,0.4)$ & 1236 & 4380 \\
IV $(700,0.5)$ & 1288 & 4452 \\
Estimates $[4,49,50]$ & $1000-1600$ & $4600-5100$ \\
\hline \hline
\end{tabular}

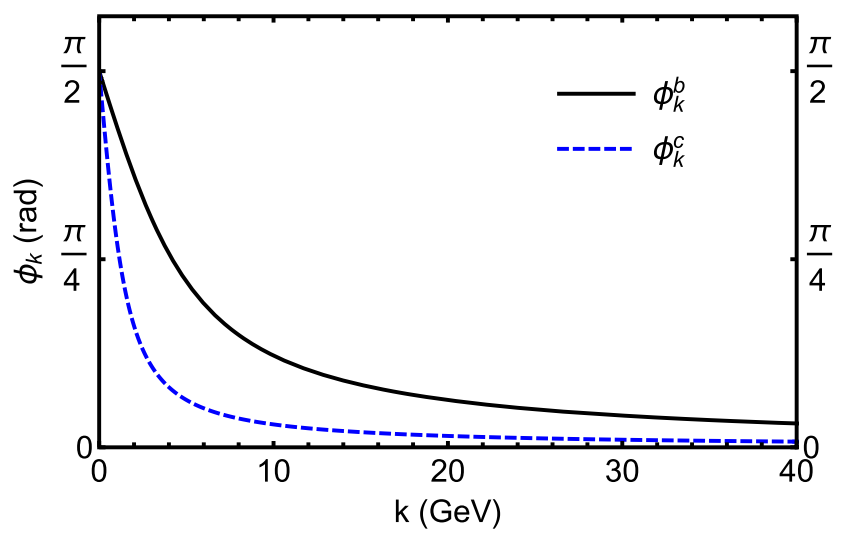

FIG. 1. Bogoliubov angles $\phi_{k}^{b}$ and $\phi_{k}^{c}$, obtained from the solutions of the gap equation [Eq. (9)], as a function of the modulus of the momentum $(k)$. We have used the current quark masses $m_{b}=4000 \mathrm{MeV}, m_{c}=950 \mathrm{MeV}$, and the set III for the parameters $\left(m_{g}, C_{h}\right)$, in conformity with Table I.

implies the finiteness of the vacuum energy. At small values of $k$, the solutions present a linear behavior with a negative slope and a sharp peak, yielding $\phi_{k}^{b, c} \rightarrow \pi / 2$ at $k \rightarrow 0$, which also assures the finite-energy density of the vacuum. Although the specific curves for $\phi_{k}^{b, c}$ obviously depend on the potentials and parameters considered, the point to be stressed is that this formalism yields well-behaved solutions of the gap equation that will be used as inputs in the obtention of the meson spectrum.

\section{A. Mass spectrum}

Now we report our predictions for the energy levels for the $B_{c}$ states, extracted from the numerical solutions of the TDA equation in Eq. (16) considering the different quantum numbers. In Table II, we list the computed masses for ground and radially excited states of $b \bar{c}$ while considering the different sets of input parameters of Table I. It gives an overall view of the behavior of computed masses as the parameters $C_{h}$ and $m_{g}$ change. In the region of parameter space considered, the augmentation of the constituent gluon mass by $100 \mathrm{MeV}$ increases the estimates by about 100-200 MeV, and strengthening the magnitude of the transverse potential by 0.1 yields masses that are about $100-150 \mathrm{MeV}$ greater.

On experimental grounds, set of parameters III seems to generate findings that better fit the observed states. Although fine-tuning of the parameters can give even better outcomes, we believe that set III is sufficient to generate findings in good conformity with the observed states. The spectrum generated for this set is shown schematically in Fig. 2.

It is also noteworthy to evaluate our predictions in light of other works existent in the literature. To this end, in Table III our calculated $B_{c}$ masses with set of parameters III 
TABLE II. TDA masses of lowest-lying and radially excited $B_{c}$ states obtained for $m_{c}=950 \mathrm{MeV}$ and $m_{b}=4000 \mathrm{MeV}$. The second column denotes the set of parameters $\left(m_{g}, C_{h}\right)$, used in conformity with Table I. The masses are given in MeV. Our calculated masses are rounded to $1 \mathrm{MeV}$. The mixing angles used are $\theta_{1 P}-\theta_{5 P}=35.3^{\circ}, \theta_{1 D}=42.5^{\circ}, \theta_{2 D}=42.2^{\circ}, \theta_{3 D}=33.2^{\circ}$, $\theta_{4 D}=21.1^{\circ}$, and $\theta_{5 D}=5.2^{\circ}$. The results that fit better to the observed states are in boldface font.

\begin{tabular}{|c|c|c|c|c|c|c|}
\hline State $\left(J^{P}\right)$ & Set & $E_{1}$ & $E_{2}$ & $E_{3}$ & $E_{4}$ & $E_{5}$ \\
\hline \multirow[t]{4}{*}{$0^{-}$} & I & 6146 & 6619 & 6986 & 7298 & 7573 \\
\hline & II & 6212 & 6733 & 7136 & 7477 & 7779 \\
\hline & I I I & 6277 & 6845 & 7284 & 7656 & 7983 \\
\hline & IV & 6417 & 6977 & 7411 & 7779 & 8103 \\
\hline \multirow[t]{4}{*}{$0^{+}$} & I & 6449 & 6852 & 7187 & 7478 & 7740 \\
\hline & II & 6545 & 6989 & 7356 & 7675 & 7961 \\
\hline & III & 6639 & 7123 & 7523 & 7871 & 8181 \\
\hline & IV & 6786 & 7264 & 7659 & 8002 & 8309 \\
\hline \multirow[t]{4}{*}{$1^{-}$} & I & 6154 & 6625 & 6990 & 7302 & 7576 \\
\hline & II & 6222 & 6739 & 7141 & 7482 & 7782 \\
\hline & III & 6288 & 6853 & 7290 & 7661 & 7988 \\
\hline & IV & 6431 & 6986 & 7418 & 7785 & 8108 \\
\hline \multirow[t]{4}{*}{$1^{+}$} & I & 6423 & 6821 & 7153 & 7443 & 7703 \\
\hline & II & 6516 & 6955 & 7318 & 7635 & 7920 \\
\hline & III & 6606 & 7088 & 7488 & 7836 & 8148 \\
\hline & IV & 6744 & 7216 & 7608 & 7950 & 8254 \\
\hline \multirow[t]{4}{*}{$1^{+\prime}$} & I & 6456 & 6845 & 7171 & 7458 & 7715 \\
\hline & II & 6552 & 6979 & 7339 & 7653 & 7935 \\
\hline & III & 6.656 & 7121 & 7513 & 7856 & 8164 \\
\hline & IV & 6783 & 7243 & 7629 & 7968 & 8272 \\
\hline \multirow[t]{4}{*}{$2^{+}$} & I & 6468 & 6853 & 7178 & 7463 & 7720 \\
\hline & II & 6568 & 6991 & 7347 & 7659 & 7940 \\
\hline & III & 6667 & 7127 & 7515 & 7854 & 8159 \\
\hline & IV & 6805 & 7259 & 7641 & 7976 & 8277 \\
\hline \multirow[t]{4}{*}{$2^{-}$} & I & 6687 & 7023 & 7331 & 7600 & 7845 \\
\hline & II & 6812 & 7177 & 7512 & 7808 & 8076 \\
\hline & III & 6931 & 7334 & 7694 & 8015 & 8306 \\
\hline & IV & 7046 & 7441 & 7815 & 8135 & 8422 \\
\hline \multirow[t]{4}{*}{$2^{-\prime}$} & I & 6687 & 7043 & 7338 & 7608 & 7853. \\
\hline & II & 6801 & 7197 & 7522 & 7816 & 8085 \\
\hline & III & 6920 & 7345 & 7704 & 8024 & 8315 \\
\hline & IV & 7068 & 7492 & 7828 & 8144 & 8432 \\
\hline
\end{tabular}

are compared with other theoretical results and available experimental data. Stated explicitly, the theoretical frameworks employed in the other studies are a relativized constituent quark model with the presence of a linear confining potential and a color Coulomb interaction [17], a constituent quark model in the heavy-quark symmetry limit with scalar confining and vector Coulomb potentials [18], a nonrelativistic quark model consisting of a confinement potential and one-gluon exchange potential [9], and a nonrelativistic linear potential model with a spin-dependent interaction [14].
We stress that our findings, specially for $B_{c}(1 S)$ and $B_{c}^{*}(2 S)$ states, present a very good fit with the measured data when the experimental errors are borne in mind. But this comparison must be done with care because the observed $B_{c}^{*}(2 S)$ peak has a mass lower than the true value, which remains unknown due to the impossibility of reconstruction of the low-energy photon emitted in the $B_{c}^{*+} \rightarrow B_{c}^{+} \gamma$, as pointed out in Ref. [6]. Moreover, the mass of the first radial excitation $B_{c}(2 S)$ is heavier than the ground state $B_{c}(1 S)$ by about $557 \mathrm{MeV}$, which is fairly good in light of experimental observations, keeping in mind the fact that $B_{c}(2 S)$ is not yet well established, according to PDG [4].

Furthermore, it can be seen that our outcomes get the $B_{c}$ spectrum in reasonable concordance with other potential model predictions. In general, the masses predicted by us for the low-lying states have a difference with respect to previous works ranging from a few $\mathrm{MeV}$ up to tens of $\mathrm{MeV}$. The exceptions having larger mass deviations are the $1^{3} P_{2}, 1 P_{1}$, and $1 P_{1}^{\prime}$ states. For higher mass states, bigger discrepancies among the predictions are evident, but most of our results are between the lower and upper values reported in Table II. In particular, our results for $1 S$-, $2 S$-, $3 S$-, and $2 P$-wave states are up to a few tens of $\mathrm{MeV}$ discrepant from those in the relativized constituent quark model of Ref. [17], while the results for $1 P_{2^{-}}, 1 P_{1}^{(\prime)}$, and $1 D$-wave states are about $100-140 \mathrm{MeV}$ smaller.

\section{B. Regge trajectories}

In addition, the energy levels listed in Tables II and III allow us to obtain the mass relation between the ground states and their radial and angular excited states, and therefore to construct the Regge trajectories in the $\left(n, M^{2}\right)$ and $\left(J, M^{2}\right)$ planes. They are then plotted in Fig. 3. In these plots, we assume that the Regge slopes are independent of charge conjugation, in accordance with the $C$ invariance of QCD [51], and also that the slopes of the parity partner trajectories coincide.

It can be remarked that the behavior of squared masses with radial quantum number and $J^{P}$ (Fig. 3, top and middle panels) is not exactly linear. This fact is clearly pronounced in the $\left(n, M^{2}\right)$ plane due to the high excitation number. More precisely, the daughter trajectories (incorporating both radially and orbitally excited states) manifest extrapolations closer to a linear fit. However, the parent trajectories (beginning from the ground states) reveal a nonlinear nature, mostly in the region of smaller mass. This is in qualitative accordance with other works that have investigated heavy quarkonia states; see, for instance, Refs. [51-55]. Notwithstanding, using the linear approximation for the Regge trajectories through the laws [52,56-58], 


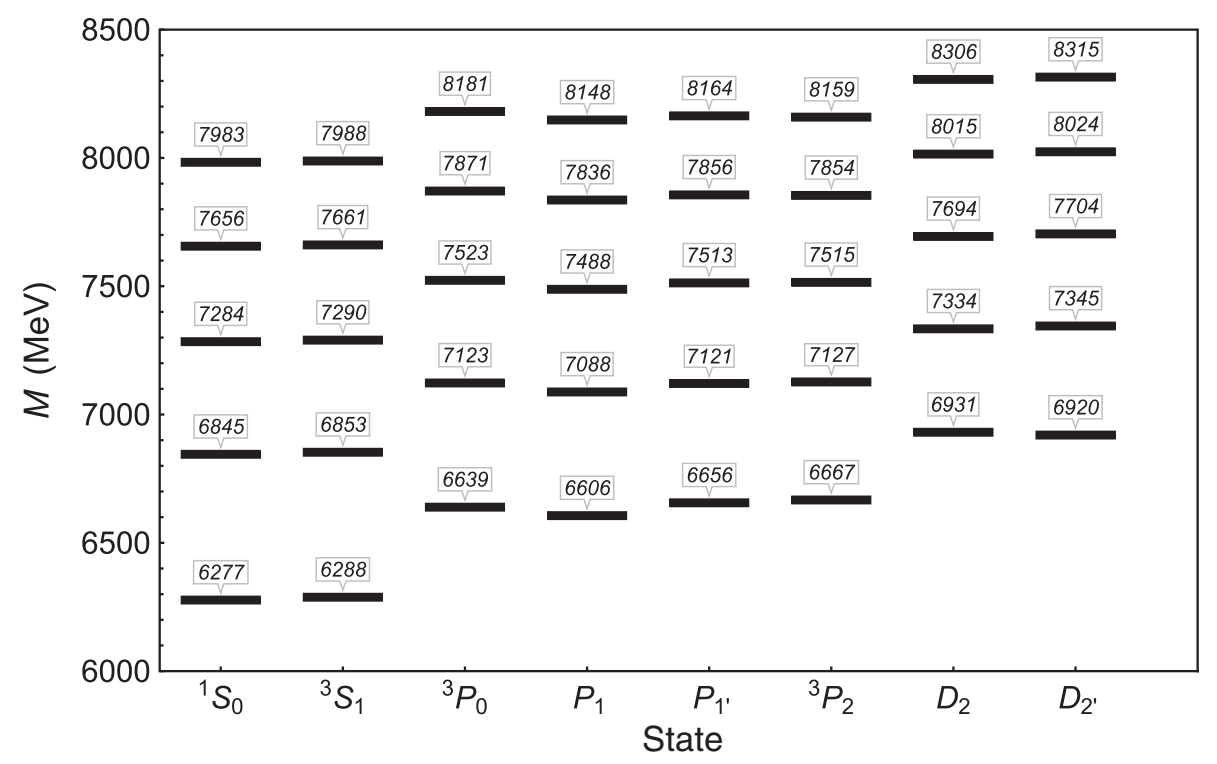

FIG. 2. $\quad B_{c}$ spectrum generated for set of parameters III.

TABLE III. (Last column) TDA masses of lowest-lying and radially excited $B_{c}$ states obtained for set of parameters III in Table I. (Other columns) Predictions of other works existent in the literature and available experimental data. $B_{c}\left(1^{1} S_{0}\right)$ is the only established particle, and its mass has been taken from PDG [4]. The states observed by ATLAS [5], CMS [6], and LHCb [7] are not considered as well established by PDG; quantum numbers of the so-called $2^{1} S_{0}$ need to be confirmed. The masses are given in MeV.

\begin{tabular}{|c|c|c|c|c|c|c|c|c|}
\hline State & $J^{P}$ & Ref. [17] & Ref. [18] & Ref. [9] & Ref. [12] & Ref. [14] & Expt. data $\left([5],{ }^{*}[6],{ }^{\dagger}[7]^{*}\right)$ & Our results (set III) \\
\hline$B_{c}\left(1^{3} S_{1}\right)$ & $1^{-}$ & 6338 & 6340 & 6357 & 6314 & 6326 & $\ldots$ & 6288 \\
\hline$B_{c}\left(1^{1} S_{0}\right)$ & $0^{-}$ & 6271 & 6260 & 6275 & 6274 & 6271 & 6275 (PDG) & 6277 \\
\hline$B_{c}\left(2^{3} S_{1}\right)$ & $1^{-}$ & 6887 & 6900 & 8697 & 6855 & 6890 & $6842,,^{\dagger} 6841^{\ddagger}$ & 6853 \\
\hline$B_{c}\left(2^{1} S_{0}\right)$ & $0^{-}$ & 6855 & 6850 & 6862 & 6841 & 6871 & $6842,6871,{ }^{\dagger} 6872^{\ddagger}$ & 6845 \\
\hline$B_{c}\left(3^{3} S_{1}\right)$ & $1^{-}$ & 7272 & 7280 & 7333 & 7206 & 7252 & $\ldots$ & 7290 \\
\hline$B_{c}\left(3^{1} S_{0}\right)$ & $0^{-}$ & 7250 & 7240 & 7308 & 7197 & 7239 & $\cdots$ & 7284 \\
\hline$B_{c}\left(1^{3} P_{2}\right)$ & $2^{+}$ & 6768 & 6760 & 6737 & 6753 & 6787 & $\cdots$ & 6667 \\
\hline$B_{c}\left(1 P_{1}^{\prime}\right)$ & $1^{+}$ & 6750 & 6740 & 6734 & 6744 & 6776 & $\cdots$ & 6656 \\
\hline$B_{c}\left(1 P_{1}\right)$ & $1^{+}$ & 6741 & 6730 & 6686 & 6725 & 6757 & $\cdots$ & 6606 \\
\hline$B_{c}\left(1^{3} P_{0}\right)$ & $0^{+}$ & 6706 & 6680 & 6638 & 6701 & 6714 & $\cdots$ & 6639 \\
\hline$B_{c}\left(2^{3} P_{2}\right)$ & $2^{+}$ & 7164 & 7160 & 7175 & 7111 & 7160 & $\ldots$ & 7127 \\
\hline$B_{c}\left(2 P_{1}^{\prime}\right)$ & $1^{+}$ & 7150 & 7150 & 7173 & 7098 & 7150 & $\cdots$ & 7121 \\
\hline$B_{c}\left(2 P_{1}\right)$ & $1^{+}$ & 7145 & 7140 & 7137 & 7105 & 7134 & $\cdots$ & 7088 \\
\hline$B_{c}\left(2^{3} P_{0}\right)$ & $0^{+}$ & 7122 & 7100 & 7084 & 7086 & 7107 & $\cdots$ & 7123 \\
\hline$B_{c}\left(3^{3} P_{2}\right)$ & $2^{+}$ & $\ldots$ & 7480 & 7575 & 7406 & 7464 & $\cdots$ & 7515 \\
\hline$B_{c}\left(3 P_{1}^{\prime}\right)$ & $1^{+}$ & $\ldots$ & 7470 & 7572 & 7393 & 7458 & $\cdots$ & 7513 \\
\hline$B_{c}\left(3 P_{1}\right)$ & $1^{+}$ & $\ldots$ & 7460 & 7546 & 7405 & 7441 & $\ldots$ & 7488 \\
\hline$B_{c}\left(3^{3} P_{0}\right)$ & $0^{+}$ & $\ldots$ & 7430 & 7492 & 7389 & 7420 & $\ldots$ & 7523 \\
\hline$B_{c}\left(1 D_{2}^{\prime}\right)$ & $2^{-}$ & 7036 & $\ldots$ & 7003 & 6984 & 7032 & $\ldots$ & 6920 \\
\hline$B_{c}\left(1 D_{2}\right)$ & $2^{-}$ & 7041 & $\cdots$ & 6974 & 6986 & 7024 & $\cdots$ & 6931 \\
\hline$B_{c}\left(2 D_{2}^{\prime}\right)$ & $2^{-}$ & $\ldots$ & $\ldots$ & 7408 & 7293 & 7347 & $\ldots$ & 7345 \\
\hline$B_{c}\left(2 D_{2}\right)$ & $2^{-}$ & $\cdots$ & $\cdots$ & 7385 & 7294 & 7343 & $\cdots$ & 7334 \\
\hline$B_{c}\left(3 D_{2}^{\prime}\right)$ & $2^{-}$ & $\cdots$ & $\ldots$ & 7783 & 7562 & 7623 & $\cdots$ & 7704 \\
\hline$B_{c}\left(3 D_{2}\right)$ & $2^{-}$ & $\cdots$ & $\cdots$ & 7781 & 7563 & 7620 & $\cdots$ & 7694 \\
\hline
\end{tabular}



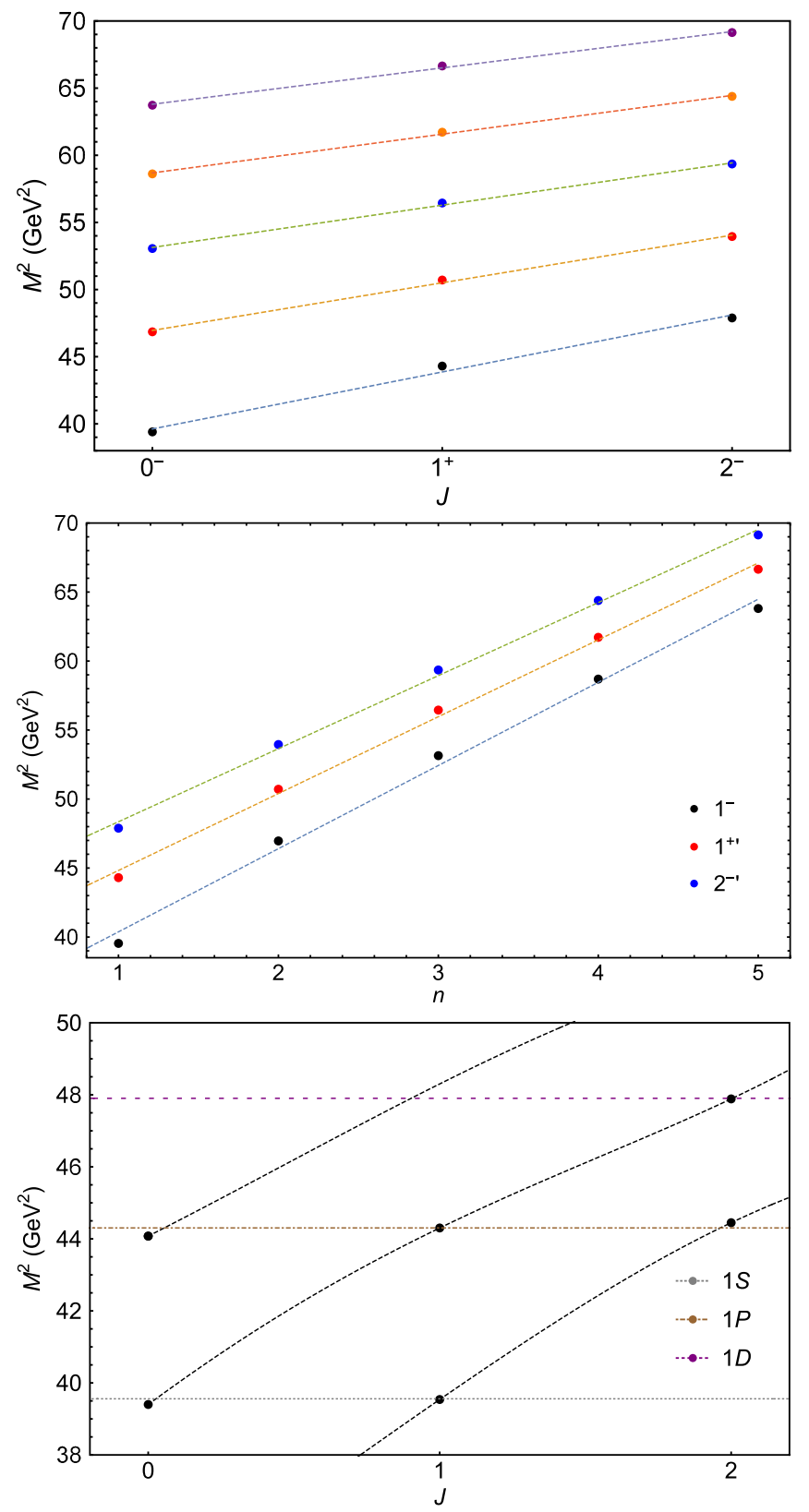

FIG. 3. Top panel: parent and Regge trajectories in the $\left(J, M^{2}\right)$ plane for $B_{c}$ states with unnatural parity $P=(-1)^{J}$ for $n=$ $1, \ldots, 5$ (from bottom to top). Middle panel: Regge trajectories in $\left(n, M^{2}\right)$ plane for $S$-wave vector, $P$-wave vector, and $D$-wave tensor $B_{c}$ states. Bottom panel: nonlinear trajectories in the $\left(J, M^{2}\right)$ plane starting from vector, pseudoscalar, and scalar $B_{c}$ states (from bottom to top), with lines indicating the regions of the $1 S, 1 P$, and $1 D$ states. Circles represent the predicted masses shown in Table II, taking the values of set of parameters III.

$$
\begin{aligned}
& M^{2}(J)=\alpha_{0} \beta+J \alpha, \\
& M^{2}(n)=\beta_{0}+n \beta,
\end{aligned}
$$

where $\alpha_{0}, \beta_{0}$ are the intercepts and $\alpha, \beta$ the slopes of each corresponding trajectory on which the meson lies.
TABLE IV. Fitted parameters for the linear fit in Eq. (19) of parent and daughter Regge trajectories in the top and middle panels of Fig. 3. The quantities are given in $\mathrm{GeV}^{2}$.

\begin{tabular}{lcc}
\hline \hline & $\left(J, M^{2}\right)$ plane & \\
\hline Trajectory & $\alpha_{0}$ & $\alpha$ \\
\hline Parent & 39.620 & 4.243 \\
First daughter & 46.956 & 3.548 \\
Second daughter & 53.137 & 3.147 \\
Third daughter & 58.689 & 2.885 \\
Fourth daughter & 63.801 & 2.705 \\
& $\left(n, M^{2}\right)$ plane & \\
State & $\beta_{0}$ & $\beta$ \\
$1^{-}$ & 34.349 & 6.027 \\
$1^{+\prime}$ & 39.253 & 5.570 \\
$2^{-}$ & 43.060 & 5.294 \\
\hline \hline
\end{tabular}

Applying this hypothesis in our scenario, we can now extract the parameters $\alpha_{0}, \beta_{0}, \alpha, \beta$ from the linear fits displayed in the top and middle panels of Fig. 3. The estimated values are listed in Table IV. These results are in reasonable accordance with the existing literature when compared, for example, with Ref. [52].

\section{Hyperfine splittings}

Additionally, another relevant feature to be noticed is the hyperfine splitting of $B_{c}$ states. We start with $S$-wave states. The hyperfine splittings $\Delta_{n S}^{\mathrm{HFS}}$ are listed in Table $\mathrm{V}$ for set of parameters III chosen as well as other sets to see the influence of their change. Despite the small values obtained for these sets, as expected, these splittings decrease for higher excited states and get larger as the parameter $C_{h}$ grows. Our calculations yield the mass for $B_{c}(2 S)$ state heavier than for $B_{c}^{*}(2 S)$ state, which coincides with the other theoretical expectations. Nonetheless, our estimations engender smaller $\Delta_{2 S}^{\mathrm{HFS}}=M\left(2^{3} S_{1}\right)-M\left(2^{1} S_{0}\right)$ hyperfine splitting, as remarked in Table $\mathrm{V}$, but not too different from the finding in Ref. [14].

Now we devote our attention to the hyperfine splitting of the $P$-wave states. For a systematic discussion of the hyperfine splitting for $P$-wave states in the context of hidden-flavor quarkonia, see, for example, Ref. [59]. In the

TABLE V. The hyperfine splittings for the $B_{c}$ system states, obtained for the set of parameters in Table I. We use the definition for the hyperfine splitting: $\Delta_{n S}^{\mathrm{HFS}}=M\left(n^{3} S_{1}\right)-M\left(n^{1} S_{0}\right)$. The set used in Table III is in boldface font. The quantities are given in $\mathrm{MeV}$.

\begin{tabular}{lccccc}
\hline \hline Set & $\Delta_{1 S}^{\mathrm{HFS}}$ & $\Delta_{2 S}^{\mathrm{HFS}}$ & $\Delta_{3 S}^{\mathrm{HFS}}$ & $\Delta_{4 S}^{\mathrm{HFS}}$ & $\Delta_{5 S}^{\mathrm{HFS}}$ \\
\hline I & 8 & 6 & 4 & 4 & 3 \\
II & 10 & 6 & 5 & 5 & 3 \\
II I & $\mathbf{1 1}$ & $\mathbf{8}$ & $\mathbf{6}$ & $\mathbf{5}$ & $\mathbf{5}$ \\
IV & 14 & 9 & 7 & 6 & 5 \\
\hline \hline
\end{tabular}


TABLE VI. Ratio $r$ defined in Eq. (21) for the lowest-lying and radially excited $P$-wave $B_{c}$ states reported in Fig. 2.

\begin{tabular}{cccccc}
\hline \hline$n$ & 1 & 2 & 3 & 4 & 5 \\
\hline$r$ & 1.0047 & 1.0031 & 1.0021 & 1.0014 & 1.0008 \\
\hline \hline
\end{tabular}

case of bottom-charmed quarkonia, let us follow as motivation the discussion provided in Ref. [15]. Experimentally, it can be remarked that the spin-singlet $P$-wave states almost coincide with the spin-averaged centroid of the triplet [4] for $c \bar{c}$ and $b \bar{b}$ systems, ideally yielding

$$
E\left(n^{1} P_{1}\right)=\frac{1}{9}\left[5 E\left(n^{3} P_{2}\right)+3 E\left(n^{3} P_{1}\right)+E\left(n^{3} P_{0}\right)\right] .
$$

Since for the $B_{c}$ system the $C$ parity is no longer a good quantum number, the states $n^{3} P_{1}-n^{1} P_{1}$ can mix according to Eq. (17), and therefore Eq. (20) cannot be directly used. Nevertheless, assuming that the relation $E\left(n^{3} P_{1}\right) \approx$ $E\left(n^{1} P_{1}\right)$ holds for $B_{c}$ mesons, Eq. (20) gives [60]

$$
E\left(n^{3} P_{0}\right)+5 E\left(n^{3} P_{2}\right)=3\left[E\left(n P_{1}\right)+E\left(n P_{1}^{\prime}\right)\right] .
$$

To test the validity of the relation above, the following ratio among the masses can be introduced [15]:

$$
r=\frac{E\left(n^{3} P_{0}\right)+5 E\left(n^{3} P_{2}\right)}{3\left[E\left(n P_{1}\right)+E\left(n P_{1}^{\prime}\right)\right]} .
$$

Thus, the deviation from $r=1$ accounts for the violation in Eq. (21). In this way, the estimations of $r$ for our calculations reported above are listed in Table VI. We see that the relative theoretical errors for the lowest-lying and radially excited states are below $0.5 \%$, and they are even smaller for the excited states. Thus, the relation in Eq. (21) holds to a fair precision. Another test that can be done is that when we augment the strength of the transverse potential, which is associated with the hyperfine interaction within the formalism used, the ratio $r$ increases (see, for example, the calculated masses for set IV in Table II). This suggests that any deviation from $r=1$ depends on the hyperfine interaction, which is in consonance with the conclusions of Ref. [15].

As a final remark, this effective approach with a small number of parameters allows us to construct the general aspects of the bottom-charmed spectrum. Our predicted energy levels are in fair agreement with other predictions, providing a guide to the experimental search for the unobserved $B_{c}$ mesons.

\section{CONCLUDING REMARKS}

The purpose of this work has been the investigation of the $B_{c}$-meson spectrum by employing a different formalism with respect to the preceding analyses. The framework employed has been an effective version of the Coulomb gauge QCD and many-body techniques associated with the TDA. The interactions between quarks (quasiparticles) and antiquarks (antiquasiparticles) have been given by the sum of an improved confining potential and a transverse hyperfine interaction with a Yukawa-type kernel, interpreted as the exchange of a constituent gluon.

Making use of a small number of parameters (dynamical mass of a constituent gluon $m_{g}$, current quark masses $m_{b}$ and $m_{c}$, and magnitude of the transverse potential $C_{h}$ ), this approach has allowed us to analyze the basic features of $B_{c}$ mesons. The calculated masses have been optimized so as to fit them to the observed states by means of tuning the parameters. Besides, the estimations of expected but yetunobserved states are approximately in accordance with other findings in the literature coming from distinct formalisms. In particular, our calculations yield a mass for $B_{c}(2 S)$ state lighter than that for $B_{c}^{*}(2 S)$ state, which coincides with the other theoretical expectations, but not with the CMS and $\mathrm{LHCb}$ results at the present moment. One expects that future findings will show that the true $B_{c}^{*}(2 S)$ peak must be at a higher mass, as the photon emitted in the $B_{c}^{*+} \rightarrow B_{c}^{+} \gamma$ radiative transition can be reconstructed.

Another aspect regarded has been the mass relation between the ground states and their radial excited states in the $\left(n, M^{2}\right)$ and $\left(J, M^{2}\right)$ planes. The nonlinearity is more pronounced in the $\left(n, M^{2}\right)$ plane due to the high excitation number used. The parent trajectories (beginning from the ground states) reveal a nonlinear nature more evident than the daughter trajectories (incorporating both radially and orbitally excited states), which is in qualitative accordance with other works exploring quarkonia states and mesons.

Further, the hyperfine splitting of both $S$ - and $P$-wave states has been studied. In both cases, we found that these splittings decrease for higher excited states but become larger as the parameter $C_{h}$ grows, as expected, since it drives the strength of the term associated with the hyperfine interaction. In the case of $P$-wave states, the mass relation in Eq. (21) has relative theoretical errors for lowest-lying and radially excited states lower than $0.5 \%$. Thus, this framework engenders a reasonable precision for this mass relation involving the $P$-wave states.

Hence, we believe that this effective approach with a minimal number of parameters is capable of offering the general aspects of the bottom-charmed spectrum, in fair agreement with other predictions. At last, all of these works provide a guide to the experimental search for the unobserved $B_{c}$ mesons. Some obvious extensions that deserve future studies are calculations and predictions on radiative and strong transitions and also on the hybrid meson spectrum.

\section{ACKNOWLEDGMENTS}

We are grateful to Felipe J. Llanes-Estrada for the support and discussions. We also would like to thank the Brazilian 
funding agencies for their financial support: CNPq (L. M. A., Contracts No. 308088/2017-4 and No. 400546/2016-7) and FAPESB (L. M. A., Contract No. INT0007/2016; F. M. C. J., Contract No. BOL2388/2017).

\section{APPENDIX A: WAVE FUNCTIONS OF TDA EQUATION OF MOTION}

In this Appendix, we present explicitly the $B_{c}$-meson spin-orbital wave functions, in terms of $L, S, J$ (helicity indices are not displayed):

(a) Pseudoscalar $(L=0, S=0, J=0)$,

$$
\psi\left(0^{-}\right)=\sqrt{\frac{1}{4 \pi}} \frac{i \sigma^{2}}{\sqrt{2}} .
$$

(b) Scalar $(L=1, S=1, J=0)$,

$$
\psi\left(0^{+}\right)=-\sqrt{\frac{1}{4 \pi}}(\boldsymbol{\sigma} \cdot \hat{k}) \frac{i \sigma^{2}}{\sqrt{2}} .
$$

(c) Vector $(L=0, S=1, J=1)$,

$$
\psi\left(1^{-}\right)=\sqrt{\frac{3}{4 \pi}} \sigma \frac{i \sigma^{2}}{\sqrt{2}} .
$$

(d) Axial $(L=1, S=0, J=1)$,

$$
\psi\left(1^{+}\right)=i \sqrt{\frac{3}{4 \pi}} \hat{\boldsymbol{k}} i \frac{i \sigma^{2}}{\sqrt{2}} .
$$

(e) Axial $(L=1, S=1, J=1)$,

$$
\psi\left(1^{+\prime}\right)=-i \sqrt{\frac{3}{8 \pi}}(\boldsymbol{\sigma} \times \hat{\boldsymbol{k}}) \frac{i \sigma^{2}}{\sqrt{2}} .
$$

(f) Tensor $(L=1, S=1, J=2)$,

$$
\psi\left(2^{+}\right)=\sqrt{\frac{3}{4 \pi}} \sigma \hat{k} \frac{i \sigma^{2}}{\sqrt{2}} .
$$

(g) Pseudotensor $(L=2, S=0, J=2)$,

$$
\psi\left(2^{-}\right)=\sqrt{\frac{5}{4 \pi}} \hat{\boldsymbol{k}} \hat{\boldsymbol{k}} \frac{i \sigma^{2}}{\sqrt{2}} .
$$

(h) Pseudotensor $(L=2, S=1, J=2)$,

$$
\psi\left(2^{-\prime}\right)=-\sqrt{\frac{5}{4 \pi}}(\boldsymbol{\sigma} \times \hat{\boldsymbol{k}}) \hat{\boldsymbol{k}} \frac{i \sigma^{2}}{\sqrt{2}} .
$$

The factor $\left(i \sigma^{2}\right)$ is introduced so as to use the same convention as Ref. [46].

\section{APPENDIX B: KERNELS OF TDA EQUATION OF MOTION}

Here we set out the relevant kernels obtained for the mesons described by the wave functions given in Eqs. (A1)-(A8):

(a) Pseudoscalar $(L=0, S=0, J=0)$,

$$
\begin{aligned}
K^{\left(0^{-}\right)}(k, q)= & V_{1}\left(a_{5}+a_{6}\right)+V_{0}\left(a_{7}+a_{8}\right) \\
& +2 U_{0}\left(a_{1}+a_{2}\right)-2 W_{0}\left(a_{3}+a_{4}\right) .
\end{aligned}
$$

(b) Scalar $(L=1, S=1, J=0)$,

$$
\begin{aligned}
K^{\left(0^{+}\right)}(k, q)= & V_{0}\left(a_{5}+a_{6}\right) \\
& +V_{1}\left(a_{7}+a_{8}\right)-2 U_{0}\left(a_{3}+a_{4}\right) \\
& +\left(U_{1}+W_{0}-k q Z_{0}\right)\left(a_{1}+a_{2}\right) .
\end{aligned}
$$

(c) Vector $(L=0, S=1, J=1)$,

$$
\begin{aligned}
K^{\left(1^{-}\right)}(k, q)= & \frac{1}{3}\left[3 V_{1}\left(a_{5}+a_{6}\right)+a_{8}\left(4 V_{2}-V_{0}\right)\right. \\
& +3 a_{7} V_{0}-2\left(a_{1}+a_{2}\right) U_{0} \\
& +2\left(a_{3}+a_{4}\right) U_{1}+2 q k\left(a_{3}+a_{4}\right) Z_{0} \\
& \left.+4\left(a_{1} k^{2}+a_{2} q^{2}\right) Z_{0}\right] .
\end{aligned}
$$

(d) Axial $(L=1, S=0, J=1)$,

$$
\begin{aligned}
K^{\left(1^{+}\right)}(k, q)= & \left(a_{5}+a_{6}\right) V_{2}+\left(a_{7}+a_{8}\right) V_{1} \\
& +2\left(a_{1}+a_{2}\right) U_{1}-2\left(a_{3}+a_{4}\right) W_{1} .
\end{aligned}
$$

(e) Axial $(L=1, S=1, J=1)$,

$$
\begin{aligned}
K^{\left(1^{+\prime}\right)}(k, q)= & \frac{1}{2}\left(V_{0}+V_{2}\right)\left(a_{5}+a_{6}\right) \\
& +\frac{1}{2}\left(U_{0}+U_{2}-2 W_{1}\right)\left(a_{3}+a_{4}\right) \\
& +V_{1}\left(a_{7}+a_{8}\right)+Z_{1}\left(a_{1} k^{2}\right. \\
& \left.+a_{2} q^{2}\right)+Z_{0} \frac{1}{2}\left(k^{2}-q^{2}\right)\left(a_{4}-a_{3}\right) .
\end{aligned}
$$


(f) Tensor $(L=1, S=1, J=2)$,

$$
\begin{aligned}
K^{\left(2^{+}\right)}(k, q)= & \frac{1}{2}\left(3 V_{2}-V_{0}\right)\left(a_{5}+a_{6}\right)+V_{1} a_{7} \\
+ & \frac{1}{5}\left(12 V_{3}-7 V_{1}\right) a_{8}+\frac{1}{5}\left(U_{1}-5 W_{0}\right. \\
& \left.-10 k q Z_{0}\right)\left(a_{1}+a_{2}\right)+\frac{12}{5} Z_{1}\left(a_{1} k^{2}+a_{2} q^{2}\right) \\
& +\frac{1}{10}\left(27 U_{2}-15 W_{1}-9 k q Z_{1}\right. \\
& \left.-4 U_{0}\right)\left(a_{3}+a_{4}\right) .
\end{aligned}
$$

(g) Pseudotensor $(L=2, S=0, J=2)$,

$$
\begin{aligned}
K^{\left(2^{-}\right)}(k, q)= & \frac{1}{2}\left(3 V_{3}-V_{1}\right)\left(a_{5}+a_{6}\right) \\
& +\frac{1}{2}\left(3 V_{2}-V_{0}\right)\left(a_{7}+a_{8}\right) \\
& +\left(3 U_{2}-U_{0}\right)\left(a_{1}+a_{2}\right) \\
& +\left(3 W_{2}-W_{0}\right)\left(a_{3}+a_{4}\right) .
\end{aligned}
$$

(h) Pseudotensor $(L=2 ; S=1 ; J=2)$,

$$
\begin{aligned}
K^{\left(2^{-\prime}\right)}(k, q)= & V_{3}\left(a_{5}+a_{6}\right)+\frac{1}{2}\left(3 V_{2}-V_{0}\right)\left(a_{7}+a_{8}\right) \\
& +k q Z_{1}\left(a_{1}+a_{2}\right) \\
& +\frac{1}{2}\left(2 Z_{2}-Z_{0}\right)\left(a_{3}+a_{4}\right) .
\end{aligned}
$$

The coefficients $a_{i}$ are given by

$$
\begin{aligned}
& a_{1}=\sqrt{1+s_{k}^{b}} \sqrt{1+s_{k}^{c}} \sqrt{1-s_{q}^{b}} \sqrt{1-s_{q}^{c}}, \\
& a_{2}=\sqrt{1-s_{k}^{b}} \sqrt{1-s_{k}^{c}} \sqrt{1+s_{q}^{b}} \sqrt{1+s_{q}^{c}}, \\
& a_{3}=\sqrt{1+s_{k}^{b}} \sqrt{1-s_{k}^{c}} \sqrt{1-s_{q}^{b}} \sqrt{1+s_{q}^{c}}, \\
& a_{4}=\sqrt{1-s_{k}^{b}} \sqrt{1+s_{k}^{c}} \sqrt{1+s_{q}^{b}} \sqrt{1-s_{q}^{c}}, \\
& a_{5}=\sqrt{1+s_{k}^{b}} \sqrt{1-s_{k}^{c}} \sqrt{1+s_{q}^{b}} \sqrt{1-s_{q}^{c}}, \\
& a_{6}=\sqrt{1-s_{k}^{b}} \sqrt{1+s_{k}^{c}} \sqrt{1-s_{q}^{b}} \sqrt{1+s_{q}^{c}}, \\
& a_{7}=\sqrt{1+s_{k}^{b}} \sqrt{1+s_{k}^{c}} \sqrt{1+s_{q}^{b}} \sqrt{1+s_{q}^{c}}, \\
& a_{8}=\sqrt{1-s_{k}^{b}} \sqrt{1-s_{k}^{c}} \sqrt{1-s_{q}^{b}} \sqrt{1-s_{q}^{c}} .
\end{aligned}
$$

The functions $s_{k(q)}^{b}$ and $s_{k(q)}^{c}$ are dependent of the gap angles obtained by solving the gap equation for the $b$ and $c$ quarks, respectively. Besides the functions $V_{n}, U_{n}$, and $W_{n}$ defined in Eqs. (10) and (11), the following auxiliary $Z$ function has been also introduced:

$$
Z(|\mathbf{k}-\mathbf{q}|) \equiv U(|\mathbf{k}-\mathbf{q}|) \frac{1-x^{2}}{|\mathbf{k}-\mathbf{q}|^{2}}
$$

[1] F. Abe et al. (CDF Collaboration), Observation of the $B_{c}$ Meson in $p \bar{p}$ Collisions at $\sqrt{s}=1.8 \mathrm{TeV}$, Phys. Rev. Lett. 81, 2432 (1998).

[2] V. Abazov et al. (D0 Collaboration), Observation of the $B_{c}$ Meson in the Exclusive Decay $B_{c} \rightarrow J / \psi \pi$, Phys. Rev. Lett. 101, 012001 (2008).

[3] R. Aaij et al. (LHCb Collaboration), Measurements of $B_{c}^{+}$ Production and Mass with the $B_{c}^{+} \rightarrow J / \psi \pi^{+}$Decay, Phys. Rev. Lett. 109, 232001 (2012).

[4] M. Tanabashi et al. (Particle Data Group), Review of particle physics, Phys. Rev. D 98, 030001 (2018).

[5] G. Aad et al. (ATLAS Collaboration), Observation of an Excited $B_{c}^{ \pm}$Meson State with the ATLAS Detector, Phys. Rev. Lett. 113, 212004 (2014).

[6] A. M. Sirunyan et al. (CMS Collaboration), Observation of Two Excited $B_{c}^{+}$States and Measurement of the $B_{c}^{+}(2 S)$ Mass in $p p$ Collisions at $\sqrt{s}=13 \mathrm{TeV}$, Phys. Rev. Lett. 122, 132001 (2019).

[7] R. Aaij et al. (LHCb Collaboration), Observation of an Excited $B_{c}^{+}$State, Phys. Rev. Lett. 122, 232001 (2019).
[8] E. J. Eichten and C. Quigg, Mesons with beauty and charm: Spectroscopy, Phys. Rev. D 49, 5845 (1994).

[9] A. P. Monteiro, M. Bhat, and K. B. Vijaya Kumar, Mass spectra and decays of ground and orbitally excited $c \bar{b}$ states in nonrelativistic quark model, Int. J. Mod. Phys. A 32, 1750021 (2017).

[10] A. P. Monteiro, M. Bhat, and K. B. Vijaya Kumar, $c \bar{b}$ spectrum and decay properties with coupled channel effects, Phys. Rev. D 95, 054016 (2017).

[11] N. R. Soni, B. R. Joshi, R. P. Shah, H. R. Chauhan, and J. N. Pandya, $Q \bar{Q}(Q \in\{b, c\})$ spectroscopy using the Cornell potential, Eur. Phys. J. C 78, 592 (2018).

[12] N. Akbar, M. Atif Sultan, B. Masud, and F. Akram, Conventional and hybrid $B_{c}$ mesons in an extended potential model, Eur. Phys. J. A 55, 82 (2019).

[13] E. J. Eichten and C. Quigg, Mesons with Beauty and Charm: New Horizons in Spectroscopy, Phys. Rev. D 99, 054025 (2019).

[14] Q. Li, M. S. Liu, L. S. Lu, Q. F. Lu, L. C. Gui, and X. H. Zhong, Excited bottom-charmed mesons in a nonrelativistic quark model, Phys. Rev. D 99, 096020 (2019). 
[15] L. Chang, M. Chen, X. Q. Li, Y. X. Liu, and K. Raya, Can the miraculous mass relation in the $P$-wave spectroscopy of charmonia and bottomonia be extended to $B_{c}$ mesons, arXiv:1912.08339.

[16] P. G. Ortega, J. Segovia, D. R. Entem, and F. Fernandez, Spectroscopy of $B_{c}$ mesons and the possibility of finding exotic $B_{c}$-like structures, Eur. Phys. J. C 80, 223 (2020).

[17] S. Godfrey and N. Isgur, Mesons in a relativized quark model with chromodynamics, Phys. Rev. D 32, 189 (1985).

[18] J. Zeng, J. W. Van Orden, and W. Roberts, Heavy mesons in a relativistic model, Phys. Rev. D 52, 5229 (1995).

[19] S. N. Gupta and J.M. Johnson, $B_{c}$ spectroscopy in a quantum chromodynamic potential model, Phys. Rev. D 53, 312 (1996).

[20] D. Ebert, R. N. Faustov, and V. O. Galkin, Properties of heavy quarkonia and $B_{c}$ mesons in the relativistic quark model, Phys. Rev. D 67, 014027 (2003).

[21] S. M. Ikhdair and R. Sever, Spectroscopy of $B_{c}$ meson in a semirelativistic quark model using the shifted large $N$ expansion method, Int. J. Mod. Phys. A 19, 1771 (2004).

[22] S. Godfrey, Spectroscopy of $B_{c}$ mesons in the relativized quark model, Phys. Rev. D 70, 054017 (2004).

[23] S. S. Gershtein, V. V. Kiselev, A. K. Likhoded, and A. V. Tkabladze, B(c) spectroscopy, Phys. Rev. D 51, 3613 (1995).

[24] T. Aliev, T. Barakat, and S. Bilmis, Properties of excited $B_{c}$ states in QCD, Nucl. Phys. B947, 114726 (2019).

[25] Z. G. Wang, Analysis of the vector and axialvector $B_{c}$ mesons with QCD sum rules, Eur. Phys. J. A 49, 131 (2013).

[26] I. F. Allison, C. T. H. Davies, A. Gray, A. S. Kronfeld, P. B. Mackenzie, and J. N. Simone, Mass of the $B_{c}$ Meson in Three-Flavor Lattice QCD, Phys. Rev. Lett. 94, 172001 (2005).

[27] R. J. Dowdall, C. T. H. Davies, T. C. Hammant, and R. R. Horgan, Precise heavy-light meson masses and hyperfine splittings from lattice QCD including charm quarks in the sea, Phys. Rev. D 86, 094510 (2012).

[28] N. Mathur, M. Padmanath, and S. Mondal, Precise Predictions of Charmed-Bottom Hadrons from Lattice QCD, Phys. Rev. Lett. 121, 202002 (2018).

[29] M. Chen, L. Chang, and Y. X. Liu, $B_{c}$ meson spectrum via Dyson-Schwinger equation and Bethe-Salpeter equation approach, Phys. Rev. D 101, 056002 (2020).

[30] A. Szczepaniak, E. S. Swanson, C. R. Ji, and S. R. Cotanch, Glueball Spectroscopy in a Relativistic Many-Body Approach to Hadron Structure, Phys. Rev. Lett. 76, 2011 (1996).

[31] S. Cotanch, A. Szczepaniak, E. Swanson, and C. Ji, QCD Hamiltonian approach for the glueball spectrum, Nucl. Phys. A631, 640C (1998).

[32] F. J. Llanes-Estrada and S. R. Cotanch, Meson Structure in a Relativistic Many-Body Approach, Phys. Rev. Lett. 84, 1102 (2000).

[33] A. P. Szczepaniak and E.S. Swanson, Coulomb gauge QCD, confinement, and the constituent representation, Phys. Rev. D 65, 025012 (2001).

[34] F. J. Llanes-Estrada and S. R. Cotanch, Relativistic manybody Hamiltonian approach to mesons, Nucl. Phys. A697, 303 (2002).
[35] N. Ligterink and E. S. Swanson, A Coulomb gauge model of mesons, Phys. Rev. C 69, 025204 (2004).

[36] F. J. Llanes-Estrada, S. R. Cotanch, A. P. Szczepaniak, and E. S. Swanson, Hyperfine meson splittings: Chiral symmetry versus transverse gluon exchange, Phys. Rev. C 70, 035202 (2004).

[37] F. J. Llanes-Estrada, P. Bicudo, and S. R. Cotanch, Oddballs and a Low Odderon Intercept, Phys. Rev. Lett. 96, 081601 (2006).

[38] A. P. Szczepaniak and P. Krupinski, Coulomb energy and gluon distribution in the presence of static sources, Phys. Rev. D 73, 034022 (2006).

[39] I. J. General, P. Wang, S. R. Cotanch, and F. J. LlanesEstrada, Light $1^{-+}$exotics: Molecular resonances, Phys. Lett. B 653, 216 (2007).

[40] P. Guo, A. P. Szczepaniak, G. Galata, A. Vassallo, and E. Santopinto, Gluelump spectrum from Coulomb gauge QCD, Phys. Rev. D 77, 056005 (2008).

[41] P. Guo, A. P. Szczepaniak, G. Galata, A. Vassallo, and E. Santopinto, Heavy quarkonium hybrids from Coulomb gauge QCD, Phys. Rev. D 78, 056003 (2008).

[42] J. M. Torres-Rincon and F. J. Llanes-Estrada, Heavy Quark Fluorescence, Phys. Rev. Lett. 105, 022003 (2010).

[43] W. Xie, L. Mo, P. Wang, and S. R. Cotanch, Coulomb gauge model for hidden charm tetraquarks, Phys. Lett. B 725, 148 (2013).

[44] P. Guo, T. Yepez-Martinez, and A. P. Szczepaniak, Charmonium meson and hybrid radiative transitions, Phys. Rev. D 89, 116005 (2014).

[45] D. Amor-Quiroz, T. Yepez-Martinez, P. Hess, O. Civitarese, and A. Weber, Low energy meson spectrum from a QCD approach based on many-body methods, Int. J. Mod. Phys. E 26, 1750082 (2017).

[46] L. M. Abreu, A. G. Favero, F. J. Llanes-Estrada, and A. G. Sánchez, Mixing and $m_{q}$ dependence of axial vector mesons in the Coulomb gauge QCD model, Phys. Rev. D 100, 116012 (2019).

[47] L. M. Abreu, F. M. da Costa Júnior, and A. G. Favero, Revisiting the tensor $J^{P C}=2^{--}$meson spectrum, Phys. Rev. D 101, 116016 (2020).

[48] H. G. Blundell, S. Godfrey, and B. Phelps, Properties of the strange axial mesons in the relativized quark model, Phys. Rev. D 53, 3712 (1996).

[49] A. T. Lytle, C. T. H. Davies, D. Hatton, G. P. Lepage, and C. Sturm, Determination of quark masses from $n_{f}=4$ lattice QCD and the RI-SMOM intermediate scheme, Phys. Rev. D 98, 014513 (2018).

[50] D. Hatton, C. Davies, B. Galloway, J. Koponen, G. Lepage, and A. Lytle, Charmonium properties from lattice QCD + QED: Hyperfine splitting, $J / \psi$ leptonic width, charm quark mass and $a_{\mu}^{c}$, arXiv:2005.01845.

[51] K. W. Wei and X. H. Guo, Mass spectra of doubly heavy mesons in Regge phenomenology, Phys. Rev. D 81, 076005 (2010).

[52] D. Ebert, R. N. Faustov, and V. O. Galkin, Spectroscopy and Regge trajectories of heavy quarkonia and $B_{c}$ mesons, Eur. Phys. J. C 71, 1825 (2011).

[53] J. K. Chen, Regge trajectories for heavy quarkonia from the quadratic form of the spinless Salpeter-type equation, Eur. Phys. J. C 78, 235 (2018). 
[54] J. K. Chen, Concavity of the meson Regge trajectories, Phys. Lett. B 786, 477 (2018).

[55] D. Jia and W. C. Dong, Regge-like spectra of excited singly heavy mesons, Eur. Phys. J. Plus 134, 123 (2019).

[56] A. V. Anisovich, V. V. Anisovich, and A. V. Sarantsev, Systematics of $q \bar{q}$ states in the $\left(n, M^{2}\right)$ and $\left(J, M^{2}\right)$ planes, Phys. Rev. D 62, 051502(R) (2000).

[57] A. V. Anisovich, C. A. Baker, C. J. Batty, D. V. Bugg, C. Hodd, H. C. Lu, V. A. Nikonov, A. V. Sarantsev, V. V. Sarantsev, and B.S. Zou, $I=0 C=+1$ mesons from 1920 to $2410 \mathrm{MeV}$, Phys. Lett. B 491, 47 (2000).
[58] S. S. Afonin, Properties of new unflavored mesons below 2.4 GeV, Phys. Rev. C 76, 015202 (2007).

[59] C. Peset, A. Pineda, and J. Segovia, $P$-wave heavy quarkonium spectrum with next-to-next-to-next-toleading logarithmic accuracy, Phys. Rev. D 98, 094003 (2018).

[60] In Ref. [15], it is argued that there should be possible hidden symmetry or an underlying principle that makes this relation valid for the $P$ level of charmonium and bottomonium systems, and also for an unequal-flavor case like the $B_{c}$ family. 